\title{
Management of Gingival Bleeding in Periodontal Patients with Domiciliary Use of Toothpastes Containing Hyaluronic Acid, Lactoferrin, or Paraprobiotics: A Randomized Controlled Clinical Trial
}

\author{
Andrea Butera ${ }^{1} \mathbb{D}$, Simone Gallo ${ }^{2, *} \mathbb{D}$, Carolina Maiorani ${ }^{1}$, Camilla Preda ${ }^{1}$, Alessandro Chiesa ${ }^{1}$, \\ Francesca Esposito ${ }^{1}$, Maurizio Pascadopoli ${ }^{2, *(D)}$ and Andrea Scribante ${ }^{2, *(D)}$ \\ 1 Unit of Dental Hygiene, Section of Dentistry, Department of Clinical, Surgical, Diagnostic and Pediatric \\ Sciences, University of Pavia, 27100 Pavia, Italy; andrea.butera@unipv.it (A.B.); \\ carolinamaiorani@outlook.it (C.M.); camilla.preda@unipv.it (C.P.); alessandro.chiesa@unipv.it (A.C.); \\ francesca.esposito@unipv.it (F.E.) \\ 2 Unit of Orthodontics and Pediatric Dentistry, Section of Dentistry, Department of Clinical, Surgical, \\ Diagnostic and Pediatric Sciences, University of Pavia, 27100 Pavia, Italy \\ * Correspondence: simone.gallo02@universitadipavia.it (S.G.); \\ maurizio.pascadopoli01@universitadipavia.it (M.P.); andrea.scribante@unipv.it (A.S.)
}

Citation: Butera, A.; Gallo, S.; Maiorani, C.; Preda, C.; Chiesa, A.; Esposito, F.; Pascadopoli, M.; Scribante, A. Management of Gingival Bleeding in Periodontal Patients with Domiciliary Use of Toothpastes Containing Hyaluronic Acid, Lactoferrin, or Paraprobiotics: A Randomized Controlled Clinical Trial. Appl. Sci. 2021, 11, 8586. https://doi.org/10.3390/app11188586

Academic Editor: Oleh Andrukhov

Received: 4 August 2021

Accepted: 14 September 2021

Published: 15 September 2021

Publisher's Note: MDPI stays neutral with regard to jurisdictional claims in published maps and institutional affiliations.

Copyright: (c) 2021 by the authors. Licensee MDPI, Basel, Switzerland. This article is an open access article distributed under the terms and conditions of the Creative Commons Attribution (CC BY) license (https:// creativecommons.org/licenses/by/ $4.0 /)$.

\begin{abstract}
Periodontitis is an irreversible oral disease causing the destruction of tooth-supporting tissues. In addition to scaling and root planing (SRP) procedures, patients should achieve a correct domiciliary oral hygiene in order to maintain a healthy status. The aim of the present study was to evaluate the efficacy of different toothpastes in reducing gingival bleeding in periodontal patients. In addition to a professional treatment of SRP, 80 patients were randomly divided into four groups according to the toothpaste assigned for the daily domiciliary use using an electric toothbrush: Group 1 (Biorepair Gum Protection), Group 2 (Biorepair Plus Parodontgel), Group 3 (Biorepair Peribioma PRO), and Group 4 (Meridol Gum Protection) (control group). After baseline (T0), patients were visited after 15 days (T1), 3 months (T2), and 6 months (T3). At each appointment, the following periodontal indexes were assessed: bleeding on probing (BoP), full-mouth bleeding score (FMBS), and modified sulcus bleeding index (mSBI). All the experimental toothpastes caused an immediate significant modification of the three clinical indexes measured, except for the control product. Biorepair Peribioma PRO, with its paraprobiotic content, was also the only toothpaste causing a prolonged effect, reducing BoP even at T3. Accordingly, both hyaluronic acid and lactoferrin appear as reliable supports for the domiciliary management of periodontal disease. In spite of this, paraprobiotics are likely to show the most important benefit thanks to their immunomodulating mechanism of action.
\end{abstract}

Keywords: dentistry; periodontitis; scaling and root planing; toothpastes; paraprobiotics; chlorhexidine; periodontology; clinical trial

\section{Introduction}

Periodontal disease is a degenerative, multifactorial, and irreversible inflammatory condition that regards tooth-supporting tissues [1,2]. Considering the multifactorial etiology, biofilm accumulation does not represent the only factor related to periodontitis, despite its main role; in fact, other risk conditions are represented by smoke, leukocyte deficits, immunosuppression, diabetes, and alteration of cytokines production [3]. Despite this, interventions aimed at removing dental plaque have the most important role [2]. Specifically, scaling and root planing (SRP) is the non-surgical gold standard therapy that respectively consists of the elimination of dental plaque/calculus and in smoothing contaminated dental roots [4]. 
However, the most relevant drawback of SRP is the bacterial recolonization of dental pockets, occurring in a short time after the treatment [5]. It is thus fundamental that clinicians correctly instruct patients in the domiciliary maintenance of the results obtained after SRP and motivate them to adhere to at-home treatment [6]. One of the most common methods is represented by the use of toothpastes with specific components that promote gingival relief and healing thanks to their antibacterial and antiplaque effect. Several products have been commercialized with this scope, including toothpastes based on chlorhexidine, sodium bicarbonate, fluoride natural extracts, or the latest trend-'biotics'. This latter group includes prebiotics (food for bacteria able to stimulate their growth), probiotics (living bacteria), and more recently, paraprobiotics (heat-inactivated bacteria, i.e., tyndallization), lysates (bacterial fragments obtained through fermentation in culture broth), and post-biotics (concentrated bacterial active metabolites).

The goal of this RCT was to test four different protocols of domiciliary oral hygiene management for periodontal patients based on the use of different toothpastes with different natural-extract components, among which were hyaluronic acid, lactoferrin, and paraprobiotics. The commercial products were chosen considering their recent introduction in the market and because of the fact that each of their active substances represented quite an innovative technology (especially the paraprobiotics), with respect to the control product traditionally used. The first null hypothesis of the study was that no intragroup difference would occur between the groups of patients assigned to the different kinds of toothpastes with regard to the effect on periodontal status. The second null hypothesis was that no intergroup difference would occur either.

\section{Materials and Methods}

\subsection{Materials}

The toothpastes tested and their features are shown in Table 1.

Table 1. List of the toothpastes tested in this study.

\begin{tabular}{cc}
\hline Product & Manufacturer \\
\hline Biorepair Gum & $\begin{array}{c}\text { Coswell SPA, 40050 } \\
\text { Protection }\end{array}$ \\
$\begin{array}{c}\text { Funo di Argelato, } \\
\text { Bologna, Italy }\end{array}$
\end{tabular}

\begin{tabular}{cc}
\hline & \\
Biorepair Plus & Coswell SPA, 40050 \\
Parodontgel & Funo di Argelato, \\
Bologna, Italy
\end{tabular}

Biorepair Plus Bologna, Italy

\section{Ingredients}

Aqua, Zinc Hydroxyapatite (microRepair ${ }^{\circledR}$ ) 15\%, Glycerin, Sorbitol, Silica, Cellulose Gum, Cocamidopropyl Betaine, Aroma, Sodium Myristoyl Sarcosinate, Sodium Methyl Cocoyl Taurate, Hamamelis Virginiana Leaf Extract, Spirulina Platensis Extract, Calendula Officinalis Flower Extract, Sodium Hyaluronate, Sodium Saccharin, Phenoxyethanol, Benzyl Alcohol, Sodium Benzoate, Potassium Sorbate, Limonene, Eugenol, Cl 16255.

Aqua, Zinc Hydroxyapatite (microRepair ${ }^{\circledR}$ ) 20\%, Glycerin, Sorbitol, Hydrated Silica, Silica, Cocamidopropyl Betaine, Cellulose Gum, Aroma, Lactoferrin, Sodium Myristoyl Sarcosinate, Sodium Methyl Cocoyl Taurate, Hamamelis Virginiana Leaf Extract, Spirulina Platensis Extract, Calendula Officinalis Flower Extract, Zinc PCA, Sodium Hyaluronate, Tocopheryl Acetate, Retinyl Palmitate, Sodium Saccharin, Phenoxyethanol, Benzyl Alcohol, Sodium Benzoate, Potassium Sorbate, Limonene, CI 77891.

Aqua, Zinc Hydroxyapatite *, Sorbitol, Glycerin, Hydrated Silica, Silica, Cocamidopropyl Betaine, Cellulose Gum, Aroma, Pistacia Lentiscus (Mastic) Gum Oil, Ascorbic Acid, Tocopheryl Acetate, Retynil Palmitate, Sodium Hyaluronate, Hamamelis Virginiana Leaf Extract, Spirulina Platensis Extract, Calendula Officinalis Flower Extract, Eucaliptus Globulus Leaf Oil, Bifidobacterium Breve *, Lactobacillus Acidophilus *, Sodium Myristoyl Sarcosinate, Sodium Methyl Cocoyl Taurate, Phenoxyethanol, Benzyl Alcohol, Sodium Benzoate, Sodium Saccharin, Potassium Sorbate, Maltodextrin, Citric Acid, Helianthus Annuus Seed Oil, BHT, Limonene, Eugenol, CI 77891, CI 73360.

Aqua, Sorbitol, Hydrated Silica, siliva Dimethyl Silylate, Hydroxyethylcellulose, Cocamidopropyl Betaine, PEG-40 Hydrogenated Castor Oil, Aroma, Sodium Gluconate, Limonene, PEG-3 Tallow Aminoproprylamine, Olafur, Stannous Fluoride, Sodium Saccharin, Potassium Hydroxide, Hydrochloric Acid, CI 74160. 


\subsection{Randomized Clinical Trial}

\subsubsection{Trial Design}

This study was conceived as a parallel-groups, randomized, active controlled, and single-center trial with a 1:1 allocation ratio. Approval was received by the Unit Internal Review Board of the Unit of Orthodontics and Pediatric Dentistry, University of Pavia, 27100 Pavia, Italy (approval number: 2020-0520).

\subsubsection{Participants}

All consecutive patients treated at the Unit of Dental Hygiene, Section of Dentistry, Department of Clinical, Surgical, Diagnostic, and Pediatric Sciences of the University of Pavia (Pavia, Italy), were assessed for eligibility and selected or excluded according to inclusion/exclusion criteria. They were engaged in June 2020 after signing an informed consent. The study lasted until December 2020, and all experimental phases were conducted in the abovementioned clinic.

The following were considered as inclusion criteria for patients: age from 18 to 70 years old, presence of teeth in the mouth (single- or multiple-rooted), diagnosis of periodontitis at stage III-grade B according to the latest Classification of Periodontal and Peri-Implant Diseases and Conditions (2017) [7], presence of bilateral pathological probing (BoP $>5 \mathrm{~mm}$ ) (at least one element per mouth side up to a maximum of 20 elements in total), and good compliance level [3]. Conversely, participants were excluded in cases of pregnancy, smoking, systemic diseases, presence of cardiac stimulator, and intake of antibiotics or anti-inflammatories in the last six months [3].

\subsubsection{Interventions and Outcomes}

During the first visit, the informed consent for the study was obtained. An experienced operator assessed periodontal bleeding indexes using a probe (UNC probe 15; Hu-Friedy, Chicago, IL, USA). Specifically, the following indexes were evaluated: bleeding on probing (BOP) [3], modified sulcus bleeding index (mSBI) [8], and full-mouth bleeding score (FMBS) [9]. A professional supra/sub-gingival oral hygiene treatment was then conducted using a piezoelectric unit (Multipiezo, Mectron S.p.a, Carasco, Italy) and Gracey curettes (Hu-Friedy, Chicago, IL); periodontal pockets were decontaminated with glycine powder (Mectron S.p.a., Carasco, Italy). At the end of these procedures, participants were instructed in domiciliary oral hygiene routine and motivated to adhere to it.

At a subsequent appointment, considered as baseline $\left(\mathrm{T}_{0}\right)$, the same procedures performed at the previous appointment were repeated, except for the professional oral hygiene. Patients were then randomized into four groups according to the toothpaste assigned for the domiciliary oral hygiene treatment, with a timing of twice a day for six months: Biorepair Gum Protection was assigned to Group 1, Biorepair Plus Parodontgel to Group 2, Biorepair Peribioma PRO to Group 3, and Meridol Gum Protection to Group 4 (this last considered as control). Participants were instructed to use an electric toothbrush for 2 min each time, along with a spongy dental floss and an interdental conic brush depending on the interproximal space. After baseline, participants were scheduled for a 6-month follow-up with an appointment after 15 days $\left(T_{1}\right), 3$ months $\left(T_{2}\right)$ and 6 months $\left(T_{3}\right)$ after baseline. At each visit, the baseline procedures were repeated (assessment of bleeding indexes and additional motivation to adhere to the oral hygiene routine using the electric toothbrush with the toothpaste assigned).

Table 2 shows the protocol of the study. 
Table 2. Experimental protocol.

\begin{tabular}{cll}
\hline Appointment & \multicolumn{1}{c}{ Procedures } \\
\hline & - $\quad$ Assessment of periodontal bleeding indexes \\
& Oral hygiene motivation and assignment of the toothpaste \\
for the home treatment: & Group 1: Biorepair Gum Protection \\
Baseline $\left(\mathrm{T}_{0}\right)$ & Group 2: Biorepair Plus Parodontgel \\
& Group 3: Biorepair Peribioma PRO \\
& Group 4: Meridol Gum Protection \\
\hline After 15 days $\left(\mathrm{T}_{1}\right)$ & - $\quad$ Reassessment of periodontal bleeding indexes \\
After 3 months $\left(\mathrm{T}_{2}\right)$ & Further oral hygiene motivation and continuation of the \\
After 6 months $\left(\mathrm{T}_{3}\right)$ & & home treatment assigned \\
\hline
\end{tabular}

\subsubsection{Sample Size}

Sample size calculation $(\alpha=0.05 ; \beta=90 \%$ ) for four independent study groups and a continuous primary endpoint was performed. Concerning the bleeding on probing (primary outcome), an expected mean of 41.28 was hypothesized, with a standard deviation of 18.13 [10]. The expected difference between the means was assumed to be 16.12; therefore, 20 patients for each group were targeted. Accordingly, 80 total participants were invited, and no one refused to participate or did not meet inclusion criteria. A total of 20 patients were assigned to Group 1 ( 8 males and 12 females, mean age 52 years old), 20 to Group 2 ( 11 males and 9 females, mean age 45 years old), 20 to Group 3 ( 7 males and 13 females, mean age 38 years old), and 20 to Group 4 ( 5 males and 15 females, mean age 41 years old).

Figure 1 shows the flowchart of the study.

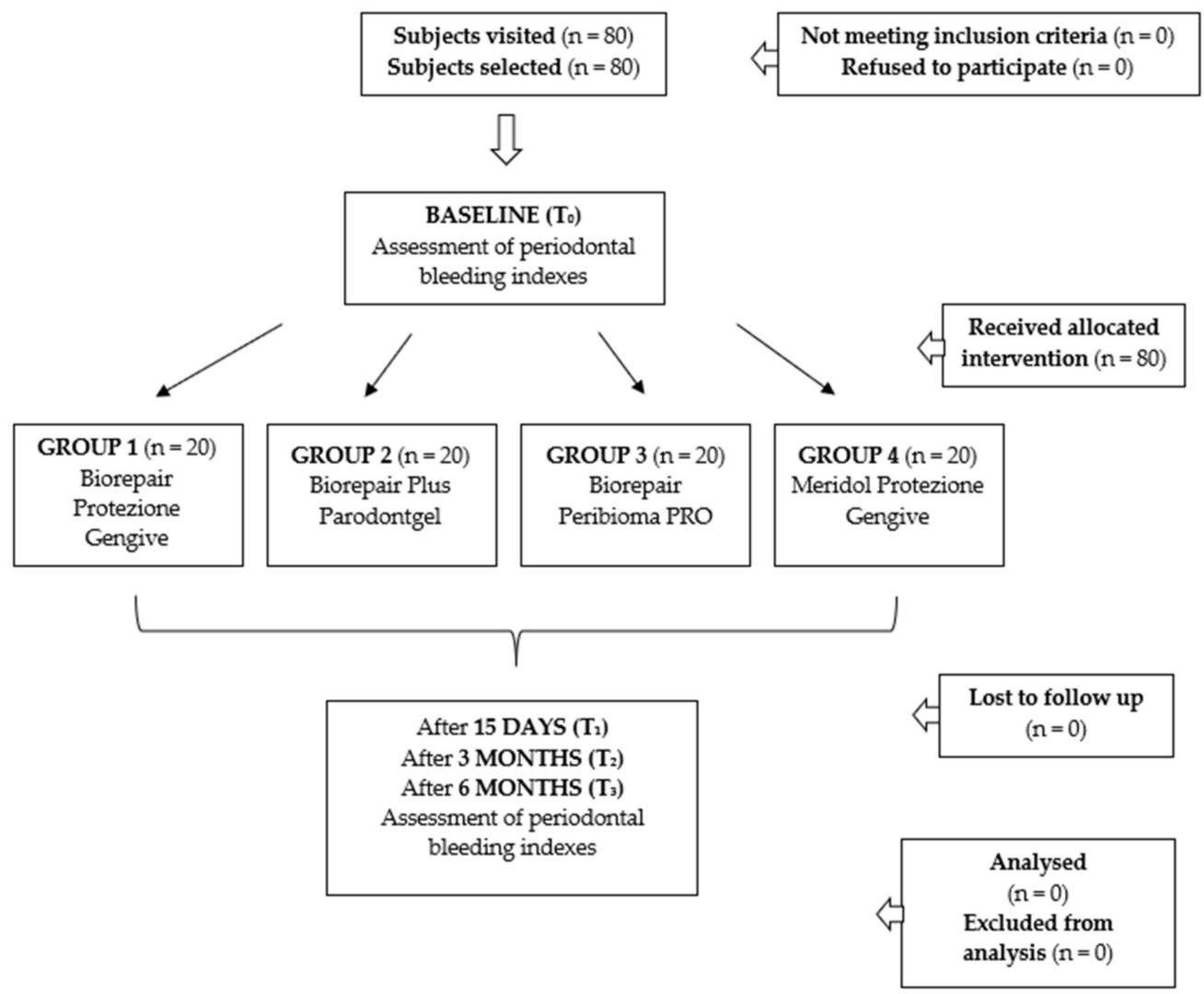

Figure 1. Flowchart of the study. 


\subsubsection{Randomization and Blinding}

Using randomization tables, the analyst provided a randomization sequence, fixing a permuted block of 20 participants. The operator who was in charge of enrolling participants also allocated them to the respective domiciliary treatment using sequentially numbered opaque and sealed envelopes with the allocation cards previously prepared; it was not technically feasible to blind him. Professional oral hygiene and data assessment were conducted by another operator. Operator/data assessor and data analyst were always blinded during the study because none of them knew which domiciliary toothpaste was assigned to each participant. Additionally, patients were asked not to reveal their respective treatment to the operator/data assessor.

\subsubsection{Statistical Methods}

Data underwent statistical analysis ( $\mathrm{R}$ software, version 3.1.3, $\mathrm{R}$ Development Core Team, R Foundation for Statistical Computing, Wien, Austria).

For each group and variable, descriptive statistics (mean and standard deviation) were calculated. As previously reported in the literature [3], bleeding on probing (BoP) was calculated as percentage, whereas full-mouth bleeding score (FMBS) and modified sulcus bleeding index (mSBI) were calculated with the relative score ranging from 0 to 3 .

The Kolmogorov-Smirnov test was used to assess data normality. For each variable, inferential comparisons between the groups were realized with ANOVA, then post hoc Tukey tests were performed. Repeated measure adjustment was performed when comparing the results of the four times for each of the four different conditions tested.

Statistical significance was set at $p<0.05$ for all the analyses conducted.

\section{Results}

\subsection{Bleeding on Probing (BoP, \%)}

As shown in Table 3, BoP significantly decreased after baseline in every group, including Group 4 (control group). No significant intergroup differences were assessed at baseline, and the highest intragroup decrease occurred in Group 2 and Group 3, whereas the lowest one occurred in the control group. No significant intragroup differences were assessed between $T_{1}$ and $T_{3}$ in any group, except in Group 3 where BoP further decreased at the last time considered.

Table 3. Descriptive statistics of bleeding on probing (BoP) index.

\begin{tabular}{|c|c|c|c|c|c|c|c|}
\hline Group & Time & Mean & St Dev & Min & Median & $\operatorname{Max}$ & Significance * \\
\hline \multirow[t]{4}{*}{$1-15 \% \mathrm{MR}$} & T0 & 91.85 & 12.42 & 55 & 100 & 100 & A \\
\hline & $\mathrm{T} 1$ & 50.75 & 13.70 & 25 & 52 & 75 & B \\
\hline & $\mathrm{T} 2$ & 47.95 & 12.77 & 23 & 49.5 & 67 & B \\
\hline & $\mathrm{T} 3$ & 44.85 & 13.39 & 20 & 43.5 & 65 & B \\
\hline \multirow[t]{4}{*}{$2-20 \% \mathrm{MR}$} & T0 & 89.45 & 14.21 & 55 & 97.5 & 100 & A \\
\hline & $\mathrm{T} 1$ & 29.75 & 8.17 & 15 & 27.5 & 50 & $\mathrm{C}$ \\
\hline & $\mathrm{T} 2$ & 28.30 & 8.89 & 13 & 28 & 48 & $\mathrm{C}$ \\
\hline & T3 & 25.55 & 8.11 & 10 & 25 & 43 & $\mathrm{C}$ \\
\hline \multirow[t]{4}{*}{$3-20 \% \mathrm{MR}+$ Paraprobiotics } & T0 & 77.00 & 21.30 & 40 & 80 & 100 & $\mathrm{~A}$ \\
\hline & $\mathrm{T} 1$ & 24.00 & 17.59 & 0 & 20 & 60 & $\mathrm{C}$ \\
\hline & $\mathrm{T} 2$ & 20.40 & 14.56 & 0 & 10 & 48 & $\mathrm{C}$ \\
\hline & T3 & 13.15 & 11.94 & 0 & 10 & 37 & $\mathrm{D}$ \\
\hline \multirow[t]{4}{*}{ 4-Control } & T0 & 81.25 & 17.23 & 50 & 80 & 100 & A \\
\hline & $\mathrm{T} 1$ & 69.25 & 18.157 & 40 & 67.5 & 100 & $\mathrm{E}$ \\
\hline & $\mathrm{T} 2$ & 68.15 & 16.468 & 43 & 69 & 98 & $\mathrm{E}$ \\
\hline & $\mathrm{T} 3$ & 57.95 & 9.8434 & 40 & 58 & 75 & $\mathrm{E}$ \\
\hline
\end{tabular}

* Different letters show statistically significant differences among the groups $(p<0.05)$. 


\subsection{Full-Mouth Bleeding Score (FMBS, 0-3)}

As shown in Table 4, FMBS significantly decreased after baseline in every group, except in Group 4 (control group), where the respective treatment assigned did not vary this index during the whole follow up. No significant intergroup differences were assessed at baseline; as for intragroup comparisons, the highest decrease between $\mathrm{T}_{0}$ and $\mathrm{T}_{1}$ occurred in Group 3, whereas a lower decrease was noticed for Group 1 and Group 2 (with no intergroup differences between the two). No significant intragroup differences were assessed between $\mathrm{T}_{1}$ and $\mathrm{T}_{3}$ in any group.

Table 4. Descriptive statistics of full-mouth bleeding score (FMBS).

\begin{tabular}{|c|c|c|c|c|c|c|c|}
\hline Group & Time & Mean & St Dev & Min & Median & $\operatorname{Max}$ & Significance * \\
\hline \multirow[t]{4}{*}{$1-15 \% \mathrm{MR}$} & T0 & 1.54 & 0.52 & 0.5 & 1.5 & 3 & A \\
\hline & $\mathrm{T} 1$ & 1.11 & 0.48 & 0.5 & 1 & 2.3 & B \\
\hline & $\mathrm{T} 2$ & 1.05 & 0.51 & 0.4 & 0.8 & 2.1 & B \\
\hline & T3 & 0.84 & 0.53 & 0.3 & 0.5 & 2 & B \\
\hline \multirow[t]{4}{*}{$2-20 \% \mathrm{MR}$} & T0 & 1.53 & 0.66 & 0.5 & 1.5 & 3 & A \\
\hline & $\mathrm{T} 1$ & 0.93 & 0.50 & 0 & 0.9 & 2 & B \\
\hline & $\mathrm{T} 2$ & 0.92 & 0.67 & 0 & 0.65 & 2 & B \\
\hline & T3 & 0.76 & 0.63 & 0 & 0.5 & 1.8 & $\mathrm{~B}$ \\
\hline \multirow[t]{4}{*}{$3-20 \% \mathrm{MR}+$ Paraprobiotics } & T0 & 1.52 & 0.56 & 0.9 & 1.4 & 2.9 & A \\
\hline & $\mathrm{T} 1$ & 0.38 & 0.33 & 0 & 0.4 & 1.2 & $\mathrm{C}$ \\
\hline & $\mathrm{T} 2$ & 0.38 & 0.21 & 0 & 0.4 & 0.8 & $\mathrm{C}$ \\
\hline & T3 & 0.26 & 0.17 & 0 & 0.2 & 0.5 & $\mathrm{C}$ \\
\hline \multirow{4}{*}{ 4-Control } & T0 & 1.83 & 0.78 & 0.5 & 2 & 3 & A \\
\hline & $\mathrm{T} 1$ & 1.675 & 0.7482 & 0.5 & 1.75 & 3 & A \\
\hline & $\mathrm{T} 2$ & 1.665 & 0.7372 & 0.8 & 1.5 & 3 & A \\
\hline & T3 & 1.49 & 0.7333 & 0 & 1.45 & 2.8 & A \\
\hline
\end{tabular}

* Different letters show statistically significant differences among the groups $(p<0.05)$.

\subsection{Modified Sulcus Bleeding Index (mSBI, 0-3)}

As shown in Table 5, mSBI significantly decreased after baseline in every group, except in Group 4 (control group), where the respective treatment assigned did not vary this index during the whole follow up. No significant intergroup differences were assessed at baseline for all the groups, as well as at all the other times in Group 1, Group 2, and Group 3.

Table 5. Descriptive statistics of modified sulcus bleeding index (mSBI).

\begin{tabular}{|c|c|c|c|c|c|c|c|}
\hline Group & Time & Mean & St Dev & Min & Median & $\operatorname{Max}$ & Significance * \\
\hline \multirow[t]{4}{*}{$1-15 \% \mathrm{MR}$} & T0 & 1.69 & 0.38 & 1 & 1.75 & 2.3 & $\mathrm{~A}$ \\
\hline & $\mathrm{T} 1$ & 1.12 & 0.49 & 0 & 1 & 2 & $\mathrm{~B}$ \\
\hline & $\mathrm{T} 2$ & 1.10 & 0.45 & 0.2 & 1 & 1.8 & B \\
\hline & T3 & 0.90 & 0.47 & 0.2 & 1 & 1.7 & $\mathrm{~B}$ \\
\hline \multirow[t]{4}{*}{$2-20 \% \mathrm{MR}$} & T0 & 1.67 & 0.66 & 0.5 & 1.8 & 3 & A \\
\hline & $\mathrm{T} 1$ & 0.83 & 0.51 & 0 & 0.9 & 2 & B \\
\hline & $\mathrm{T} 2$ & 0.72 & 0.57 & 0 & 0.65 & 2 & $\mathrm{~B}$ \\
\hline & T3 & 0.53 & 0.51 & 0 & 0.5 & 1.8 & $\mathrm{~B}$ \\
\hline \multirow[t]{4}{*}{$3-20 \% \mathrm{MR}+$ Paraprobiotics } & T0 & 1.96 & 0.76 & 0 & 2 & 3 & A \\
\hline & $\mathrm{T} 1$ & 0.84 & 0.63 & 0 & 0.8 & 2.75 & B \\
\hline & $\mathrm{T} 2$ & 0.82 & 0.74 & 0 & 0.65 & 2.54 & B \\
\hline & T3 & 0.60 & 0.62 & 0 & 0.55 & 2.3 & B \\
\hline \multirow{4}{*}{ 4-Control } & T0 & 2.03 & 0.78 & 1 & 1.8 & 3 & A \\
\hline & $\mathrm{T} 1$ & 1.85 & 0.7037 & 1 & 1.6 & 3 & $\mathrm{~A}$ \\
\hline & $\mathrm{T} 2$ & 1.835 & 0.6907 & 0.7 & 1.65 & 3 & A \\
\hline & T3 & 1.67 & 0.6449 & 0.6 & 1.5 & 2.8 & A \\
\hline
\end{tabular}




\section{Discussion}

Infections occurring in the oral cavity represent one of the most frequent concerns in dental clinical practice, and many strategies have been proposed to avoid this outcome, such as the use of anti-infective materials [11]. In addition to the colonization of teeth by cariogenic bacteria resulting in carious lesions, specific microorganisms can attack the periodontal tissues. In the presence of poor immune defenses and other eventual risk factors (such as improper oral hygiene or the habit of smoking), bacterial colonization can advance until causing a dysbiosis leading to periodontal disease [12]. Accordingly, it is fundamental for patients to properly carry out correct domiciliary oral hygiene maneuvers aimed at preventing this progression. In case this condition should occur, the conventional treatment for periodontal patients is based on a mechanical therapy (scaling and root planing, SRP) which is aimed at removing plaque/calculus and smoothing infected dental roots surfaces [4]. However, the management of periodontal disease cannot be simply based solely on professional treatment; patients must be directly involved in the therapeutic protocol, thus performing correct domiciliary hygiene procedures with specific products, as prescribed by the clinician [13]. On the basis of this consideration, a correct oral hygiene routine appears to be a fundamental preventive procedure, both to avoid the onset of periodontitis as well as to avoid a relapse when the pathology is under control.

This is particularly true especially for patients at high risk of periodontal inflammation, such as orthodontic patients or those having a periodontitis not yet diagnosed or those with predisposing systemic diseases [14]. Accordingly, the aim of the present randomized clinical trial was to evaluate different toothpastes used as a domiciliary means to pursue a good oral hygiene status in periodontal patients undergoing recurrent treatments of SRP. Specifically, the purpose was to assess the effect of the different toothpastes on the management of gingival bleeding. Accordingly, only bleeding indexes were chosen as outcomes of the present research.

The first null hypothesis of the study was rejected, whereas the second one was partially rejected. All the experimental toothpastes tested (i.e., Biorepair Gum Protection, Biorepair Plus Parodontgel, and Biorepair Peribioma PRO) resulted in being highly effective in varying all three of the periodontal bleeding indexes considered. The three agents equally modified the modified sulcus bleeding index (mSBI), with a significant reduction in this parameter between the baseline appointment and the following visit after 15 days. No further changes were detected during the subsequent follow up at 3 and 6 months; therefore, the effect of the toothpastes was exerted mainly in the short-term of daily use and was maintained unaltered at least for the six months of follow up here considered. With regard to the full-mouth bleeding score (FMBS), the toothpastes Biorepair Gum Protection and Biorepair Plus Parodontgel significantly reduced this index between $T_{0}$ and $T_{1}$, with no further reductions after this last time. In addition, no significant intergroup differences were noticed between the two abovementioned agents. However, it was Biorepair Peribioma PRO that showed the best effect with respect to the other experimental products by strongly reducing FMBS after baseline; here again, this outcome was maintained during the whole follow-up, with no variations in the meantime. As to bleeding on probing $(\mathrm{BoP})$, all the three pastes were again successful in reducing this index, especially Biorepair Plus Parodontgel and Biorepair Peribioma PRO. As previously reported for the other variables, the effect was seen immediately after the baseline time, whereas it remained unaltered subsequently. Carrying out an intergroup comparison between the last two abovementioned products, no differences for BoP were assessed at any time, except at $\mathrm{T}_{3}$ for Biorepair Peribioma PRO when the index had further decreased.

The benefit of the periodontal status obtained after using the three experimental toothpastes was directly linked to the properties of the ingredients contained inside. On the contrary, the absence of particular curative components in the control toothpaste (Meridol Gum Protection) justified the reduced benefit obtained with this product, which was limited to a slight reduction in BoP after baseline, with no influence on FMBS and mSBI. However, 
it is important to say that the clinical variations assessed cannot be connected only to the domiciliary treatment alone but are mainly influenced by the in-office SRP.

Specifically focusing on the favorable effects of the three experimental toothpastes on bleeding indexes, it is worth discussing their specific compositions to explain their action. All these experimental agents are composed of hyaluronic acid, which is a natural polysaccharide constituting the extracellular matrix of the connective tissue, and the use of which is widely recognized for the treatment of chronic periodontitis [15]. The use of this substance covers several medical areas, especially dermatology and plastic surgery; with regard to dentistry, and specifically periodontology, hyaluronic acid has anti-inflammatory, regenerative, and anti-bacterial effects with potential in the treatment of periodontal disease. Because of its tissue healing properties, this compound is used as an adjunct to mechanical therapy in the treatment of periodontitis [16]. In addition to hyaluronic acid, the potential of using the three experimental toothpastes may also be linked to medicinal plant extracts (e.g., Hamamelis virginiana Leaf Extract, Spirulina platensis Extract, and Calendula Officinalis Flower Extract) that are common among the three pastes and whose action against anaerobic and facultative aerobic periodontal bacteria has been suggested in several studies [17-19]. In particular, the effect of Calendula officinalis on oxidative stress and bone loss has been studied in rats subjected to experimental periodontitis, and a positive outcome was assessed for this substance with preservation of collagen fibers [20]. Considering Spirulina platensis Extract, it is known to have an antimicrobial effect against $S$. aureus, E. coli, P. aeruginosa, Klebsiella sp., Proteus sp., and Embeddobacter sp.; a mass of evidence is in favor of Spirulina due to its anti-inflammatory effects [21].

Despite the general change in periodontal bleeding indexes assessed after the use of the three products in the respective groups, the effect size appeared to be the same only on mSBI, whereas the change generated on BoP and FMBS appeared to be different. For instance, Biorepair Plus Parodontgel and Biorepair Peribioma PRO showed the highest impact on BoP index, whereas Biorepair Gum Protection reported a lower effect, although more significant with respect to Meridol Gum Protection. In particular, the different result observed for Biorepair Plus Parodontgel and Biorepair Gum Protection can be ascribed to the presence of lactoferrin only in the former product. The anti-inflammatory action of this substance has widely been studied in in vitro periodontitis models as well as in observational clinical studies: in vitro lactoferrin exerts strong anti-inflammatory properties against gingival fibroblasts infected with Prevotella intermedia, whereas in vivo the topical administration to patients suffering from periodontitis is able to decrease both the levels of cytokines in crevicular fluid (e.g., IL-6), as well as edema, bleeding, pocket depth, and gingival and plaque index, with a positive variation of clinical attachment levels [22] Furthermore, both Biorepair Plus Parodontgel and Biorepair Peribioma PRO contain tocopheryl acetate (vitamin E), an antioxidant agent whose positive role in periodontitis has been suggested but little studied until now. In particular, experiments conducted in rats suggest that vitamin E supplements prevent oxidative stress related to periodontitis; however, the potential for the treatment of human periodontal disease still remains uncertain because decreases in alveolar bone loss have not always been observed [23].

The most interesting results obtained in the present report are certainly the ones related to Biorepair Peribioma PRO toothpaste, which was the most effective in reducing FMBS. In addition to that, this product significantly reduced BoP index with no intergroup differences with respect to Biorepair Plus Parodontgel, except at the 6-month evaluation. In fact, in the current study, Biorepair Peribioma PRO was the only product causing not only an immediate positive effect between $T_{0}$ and $T_{1}$ but also a further delayed benefit assessed after a 6-month follow-up. No other intragroup differences between $T_{1}$ and $T_{3}$ were assessed for the other pastes tested.

Biorepair Peribioma PRO was the only paraprobiotics-based toothpaste tested in this study, specifically containing heat-inactivated bacteria (tyndallization) of the genus Lactobacillus and Bifidobacterium. Among the 'biotic' compounds, one of the most important categories is represented by probiotics. These are defined by the Food and Agriculture Orga- 
nization (FAO) and the World Health Organization (WHO) as 'live microorganisms which when administered in adequate amounts confer a health benefit on the host' [24]. Several mechanisms have been proposed to explain the beneficial effect of probiotics, including the exclusion of pathogenetic bacteria and the competition with pathogenetic bacteria for nutrients and epithelial cell adhesion, the production of antimicrobial substances against these harmful microorganisms, the enhancement of the mucosal barrier function, and the reestablishment of the physiological immunomodulatory function of the host [25]. According to a recent study by our group, probiotics are a significant support to SRP in the treatment of periodontitis, with a positive variation on periodontal clinical parameters and a reduction in specific periodontal bacteria; additionally, it has been suggested that probiotics not only reduce microbic counts but, above all, exert an immunomodulatory action, thus switching from a pro-inflammatory towards an anti-inflammatory response [3].

Despite the definition of probiotics requiring that microorganisms must be alive in order to guarantee benefits to the hosts' health, recent studies have shown that inactivated probiotic microorganisms (paraprobiotics) can provide these benefits too. For example, this 'biotic' category is likely to modulate the immune system and to secrete metabolites by the dead cells with an additional benefit [26].

The actions that paraprobiotics probably exert perfectly fit with the consideration of periodontitis as a process of tissues' impairment following the action of inflammatory and immune cells. On the basis of this consideration, it might be supposed that the modulatory function of paraprobiotics on specific periodontal cells justifies the additional benefit found in this study for the group assigned to the use of Biorepair Peribioma PRO. Additionally, as conventional antimicrobial agents might be effective in a short time frame, the different mechanism of action of paraprobiotics might guarantee a prolonged action, thus justifying the further change assessed in this study at the end of the follow-up for Biorepair Peribioma PRO. In fact, whereas the other toothpastes tend to show a more evident effect after a short-term period because of the shock-treatment exerted by the curative ingredients contained, conversely paraprobiotics are likely to be more effective in the long term. With the introduction of probiotics and paraprobiotics in dentistry, the goal of the therapeutic chemical substances, in addition to the mechanical therapy [27], has completely changed, passing from pure antimicrobial substances towards immunomodulators. Further studies should be carried out to assess which specific composition of paraprobiotics report the most beneficial action. For instance, other bacterial strains deserve to be evaluated. Finally, due to the remineralizing effect of the zinc hydroxyapatite contained in the products, the action of the tested experimental toothpastes deserves to be evaluated also in lesions occurring in patients under active orthodontic treatment [28].

The major limitation of this study is that only bleeding indexes were considered. Subsequent clinical evaluations should take into account the effects of the same products on other periodontal indexes, especially probing pocket depth (PPD) and clinical attachment level (CAL). Regarding the external validity of the study, it is not possible to generalize the results here obtained, neither to gingivitis nor to other stages/grades of periodontitis, except to those considered in our protocol. Moreover, confounding factors such as sex and age should also be considered, as well as the toothbrushes' bristles. With regard to this latter point, in fact, the kind and the texture of the bristles could in part affect the results of the present study, due to their different action on both soft and hard tissues as well as dental materials [29].

Another limitation is that the composition of bacterial colonies in the periodontal sulcus should be quantified and compared before, during, and at the end of the follow up. Finally, focusing on the better outcome obtained after employing the toothpaste with paraprobiotics, its specific mechanism of action should be further investigated, even from a microbiological and immunological point of view. 


\section{Conclusions}

Despite their heterogeneous composition, the three experimental toothpastes tested in this study (Biorepair Gum Protection, Biorepair Plus Parodontgel, and Biorepair Peribioma PRO) served a valuable role in the domiciliary management of oral hygiene in periodontal patients undergoing SRP treatment. No relevant outcomes were obtained considering Meridol Gum Protection.

On the basis of these preliminary considerations, it might be speculated that both hyaluronic acid and lactoferrin can be used for the domiciliary management of periodontal disease in addition to professional mechanical therapy. However, paraprobiotics are likely to show the most import benefit thanks to their different and innovative mechanism of action based on immunomodulation. Further laboratory and clinical investigations should be conducted to confirm these results.

Author Contributions: Conceptualization, A.B. and A.S.; methodology, A.B.; software, A.S.; validation, A.B., S.G., and A.S.; formal analysis, A.B.; investigation, A.C.; resources, C.M.; data curation, C.P.; writing-original draft preparation, S.G. and M.P.; writing—review and editing, S.G., A.B., and A.S.; visualization, A.B. and F.E.; supervision, A.S.; project administration, A.B. All authors have read and agreed to the published version of the manuscript.

Funding: This research received no external funding.

Institutional Review Board Statement: The study was conducted according to the guidelines of the Declaration of Helsinki, and the study was approved by the Internal Review Board (number of approval: 2020-0520).

Informed Consent Statement: Informed consent was obtained from all participants involved in the study.

Data Availability Statement: The data presented in this study are available on request from the corresponding author.

Acknowledgments: The authors would like to thank the manufacturers of the products tested.

Conflicts of Interest: The authors declare no conflict of interest.

\section{References}

1. Baelum, V.; Lopez, R. Periodontal disease epidemiology_Learned and unlearned? Periodontology 2000 2013, 62, 37-58. [CrossRef] [PubMed]

2. Checchi, V.; Pascolo, G. Microbiological Response to Periodontal Therapy: A Retrospective Study. Open Dent. J. 2018, 12, 837-845. [CrossRef] [PubMed]

3. Butera, A.; Gallo, S.; Maiorani, C.; Molino, D.; Chiesa, A.; Preda, C.; Esposito, F.; Scribante, A. Probiotic Alternative to Chlorhexidine in Periodontal Therapy: Evaluation of Clinical and Microbiological Parameters. Microorganisms 2021, 9, 69. [CrossRef] [PubMed]

4. Berezow, A.B.; Darveau, R.P. Microbial shift and periodontitis. Periodontology 2000 2011, 55, 36-47. [CrossRef]

5. Mombelli, A. Microbial colonization of the periodontal pocket and its significance for periodontal therapy. Periodontology 2000 2018, 76, 85-96. [CrossRef] [PubMed]

6. Checchi, L.; Montevecchi, M.; Gatto, R.M.; Moreschi, A.; Checchi, V. Clinical efficacy of two toothbrushes with different bristles. Int. J. Dent. Hyg. 2007, 5, 242-246. [CrossRef]

7. Berglundh, T.; Armitage, G.; Araujo, M.G.; Avila-Ortiz, G.; Blanco, J.; Camargo, P.M.; Chen, S.; Cochran, D.; Derks, J.; Figuero, E.; et al. Peri-implant diseases and conditions: Consensus report of workgroup 4 of the 2017 World Workshop on the Classification of Periodontal and Peri-Implant Diseases and Conditions. J. Clin. Periodontol. 2018, 45, S286-S291. [CrossRef]

8. Mombelli, A.; van Oosten, M.A.; Schurch, E., Jr.; Land, N.P. The microbiota associated with successful or failing osseointegrated titanium implants. Oral Microbiol Immunol. 1987, 2, 145-151. [CrossRef]

9. Bertoldi, C.; Forabosco, A.; Lalla, M.; Generali, L.; Zaffe, D.; Cortellini, P. How Intraday Index Changes Influence Periodontal Assessment: A Preliminary Study. Int. J. Dent. 2017, 2017, 7912158. [CrossRef] [PubMed]

10. Mühlemann, H.R.; Son, S. Gingival sulcus bleeding-A leading symptom in initial gingivitis. Helv. Odontol. Acta 1971, 15, 107-113.

11. Farhadian, N.; Bidgoli, M.; Jafari, F.; Mahmoudzadeh, M.; Yaghobi, M.; Miresmaeili, A. Comparison of Electric Toothbrush, Persica and Chlorhexidine Mouthwashes on Reduction of Gingival Enlargement in Orthodontic Patients: A Randomised Clinical Trial. Oral Health Prev Dent. 2015, 13, 301-307. 
12. Scribante, A.; Poggio, C.; Gallo, S.; Riva, P.; Cuocci, A.; Carbone, M.; Arciola, C.R.; Colombo, M. In Vitro Re-Hardening of Bleached Enamel Using Mineralizing Pastes: Toward Preventing Bacterial Colonization. Materials 2020, 13, 818. [CrossRef]

13. Deng, Z.L.; Szafrański, S.P.; Jarek, M.; Bhuju, S.; Wagner-Döbler, I. Dysbiosis in chronic periodontitis: Key microbial players and interactions with the human host. Sci. Rep. 2017, 7, 3703-3715. [CrossRef] [PubMed]

14. Costa, F.O.; Cortelli, S.C.; Costa, A.A.; Cyrino, R.M.; Cortelli, J.R.; Miranda Cota, L.O. Impact of compliance during periodontal maintenance therapy on oral health-related quality of life: A 6-year follow-up. J. Dent. 2019, 83, 50-55. [CrossRef]

15. Cosola, S.; Giammarinaro, E.; Genovesi, A.M.; Pisante, R.; Poli, G.; Covani, U.; Marconcini, S. A short-term study of the effects of ozone irrigation in an orthodontic population with fixed appliances. Eur. J. Paediatr. Dent. 2019, 20, 15-18. [PubMed]

16. Casale, M.; Moffa, A.; Vella, P.; Sabatino, L.; Capuano, F.; Salvinelli, B.; Lopez, M.A.; Carinci, F.; Salvinelli, F. Hyaluronic acid: Perspectives in dentistry. A systematic review. Int. J. Immunopathol. Pharmacol. 2016, 29, 572-582. [CrossRef] [PubMed]

17. Dahiya, P.; Kamal, R. Hyaluronic Acid: A boon in periodontal therapy. N. Am. J. Med. Sci. 2013, 5, 309-315. [CrossRef] [PubMed]

18. Iauk, L.; Lo Bue, A.M.; Milazzo, I.; Rapisarda, A.; Blandino, G. Antibacterial activity of medicinal plant extracts against periodontopathic bacteria. Phytother. Res. 2003, 17, 599-604. [CrossRef]

19. Mahendra, J.; Mahendra, L.; Muthu, J.; John, L.; Romanos, G.E. Clinical effects of subgingivally delivered spirulina gel in chronic periodontitis cases: A placebo controlled clinical trial. J. Clin. Diagn. Res. 2013, 7, 2330-2333. [CrossRef]

20. Palombo, E.A. Traditional Medicinal Plant Extracts and Natural Products with Activity against Oral Bacteria: Potential Application in the Prevention and Treatment of Oral Diseases. Evid. Based Complement. Alternat. Med. 2011, 2011, 680354. [CrossRef]

21. Maniyar, R.; Umashankar, G.K. Effectiveness of Spirulina Mouthwash on Reduction of Dental Plaque and Gingivitis: A Clinical Study. Int. J. Pharm. Pharm. Sci. 2017, 9, 136-139. [CrossRef]

22. Lima, M.D.R.; Lopes, A.P.; Martins, C.; Brito, G.A.C.; Carneiro, V.C.; Goes, P. The Effect of Calendula officinalis on Oxidative Stress and Bone Loss in Experimental Periodontitis. Front. Physiol. 2017, 8, 440. [CrossRef]

23. Berlutti, F.; Pilloni, A.; Pietropaoli, M.; Polimeni, A.; Valenti, P. Lactoferrin and oral diseases: Current status and perspective in periodontitis. Ann. Stomatol. 2011, 2, 10-18.

24. Varela-López, A.; Navarro-Hortal, M.D.; Giampieri, F.; Bullón, P.; Battino, M.; Quiles, J.L. Nutraceuticals in Periodontal Health: A Systematic Review on the Role of Vitamins in Periodontal Health Maintenance. Molecules 2018, 23, 1226. [CrossRef]

25. Joint FAO/WHO Working Group. Working Group Report on Drafting Guidelines for the Evaluation of Probiotics in Food; Joint FAO/WHO Working Group: London, ON, Canada, 2002.

26. İnce, G.; Gürsoy, H.; İpçi, Ş.D.; Cakar, G.; Emekli-Alturfan, E.; Yılmaz, S. Clinical and Biochemical Evaluation of Lozenges Containing Lactobacillus reuteri as an Adjunct to Non-Surgical Periodontal Therapy in Chronic Periodontitis. J. Periodontol. 2015, 86, 746-754. [CrossRef]

27. De Almada, C.N.; Almada, C.N.; Martinez, R.C.R.; Sant'Ana, A.S. Paraprobiotics: Evidences on their ability to modify biological responses, inactivation methods and perspectives on their application in foods. Trends Food Sci. Technol. 2016, 58, 96-114. [CrossRef]

28. Scribante, A.; Gallo, S.; Celmare, R.L.; D'Antò, V.; Grippaudo, C.; Gandini, P.; Sfondrini, M.F. Orthodontic debonding and tooth sensitivity of anterior and posterior teeth. Angle Orthod. 2020, 90, 766-773. [CrossRef] [PubMed]

29. Preda, C.; Butera, A.; Pelle, S.; Pautasso, E.; Chiesa, A.; Esposito, F.; Oldoini, G.; Scribante, A.; Genovesi, A.; Cosola, S. The Efficacy of Powered Oscillating Heads vs. Powered Sonic Action Heads Toothbrushes to Maintain Periodontal and Peri-Implant Health: A Narrative Review. Int. J. Environ. Res. Public Health 2021, 18, 1468. [CrossRef] [PubMed] 